\title{
Associação entre métodos de adaptabilidade e estabilidade em milho
}

\author{
Association between adaptability and stability methods in corn
}

\author{
Alberto Cargnelutti Filho ${ }^{\text {I* }}$ Lindolfo Storck ${ }^{\mathrm{I}}$ João Riboldi ${ }^{\mathrm{II}}$ José Paulo Guadagnin ${ }^{\mathrm{III}}$
}

\section{RESUMO}

Neste trabalho, foram usados dados de produtividade de grãos oriundos de 65 ensaios de competição de cultivares de milho, realizados no Estado do Rio Grande do Sul, nos anos agrícolas 2002/2003, 2003/2004 e 2004/2005, com o objetivo de comparar os métodos de análise de adaptabilidade e estabilidade de: Yates \& Cochran, Plaisted \& Peterson, Wricke, Annicchiarico, Finlay \& Wilkinson, Eberhart \& Russell, Tai, Toler, Silva \& Barreto, Cruz et al., Huehn, Lin \& Binns modificado por Carneiro e a análise AMMI. Para verificar o grau de associação entre as estimativas dos parâmetros de adaptabilidade e estabilidade, obtidas pelos diferentes métodos, foi utilizado o coeficiente de correlação de Spearman. Há concordância na indicação de cultivares de milho pelos métodos de Plaisted \& Peterson e Wricke, de Annicchiarico e Lin \& Binns modificado por Carneiro, de Eberhart \& Russell e Tai e de Toler e Silva \& Barreto, o que contra-indica seu uso concomitante. Em menor grau de associação, a análise AMMI concorda com as estimativas dos parâmetros de estabilidade de Plaisted \& Peterson, Wricke, Eberhart \& Russell, Tai, Silva \& Barreto e Cruz et al.

Palavras-chave: Zea mays, interação genótipos $x$ ambientes, indicação de cultivares.

\section{ABSTRACT}

Grain yield data were used from 65 maize cultivar trials carried out in the State of the Rio Grande do Sul, Brazil, in the years of 2002/2003, 2003/2004 and 2004/2005, with the objective of comparing the following methods of adaptability and stability analysis: Yates and Cochran, Plaisted and Peterson, Wricke, Annicchiarico, Finlay and Wilkinson, Eberhart and Russell, Tai, Toler, Silva and Barreto, Cruz et al., Huehn, Lin and Binns modified by Carneiro and analysis AMMI. To verify the degree of association between the estimates of adaptability and stability parameters, obtained by the different methods, the Spearman correlation coefficient was used. There is an agreement in the indication of maize cultivars by the methods of Plaisted and Peterson and Wricke, of Annicchiarico and Lin and Binns modified by Carneiro, of Eberhart and Russell and Tai, and of Toler and Silva and Barreto, which counter-indicates their concomitant use. To a lesser degree of association the AMMI analysis agrees with the estimates of the stability parameters, Plaisted and Peterson, Wricke, Eberhart and Russell, Tai, Silva and Barreto and Cruz et al.

Key words: Zea mays, genotype $x$ environment interaction, cultivar indication.

\section{INTRODUÇÃO}

$\mathrm{Na}$ fase final de um programa de melhoramento de plantas, o desempenho de cultivares em vários ambientes (locais, anos e épocas) tem sido avaliado para verificar o seu comportamento diferencial face às variações ambientais. Este comportamento diferencial é atribuído à interação genótipos x ambientes (GxA) e a sua existência exige estudo, por meio da análise de adaptabilidade e estabilidade, para garantir maior segurança às indicações de cultivares (CRUZ \& REGAZZI, 1997; CRUZ \& CARNEIRO, 2003).

Para a avaliação da adaptabilidade e da estabilidade de genótipos, existem várias metodologias que são complementares à análise de variância

'Departamento de Fitotecnia, Centro de Ciências Rurais (CCR), Universidade Federal de Santa Maria (UFSM), 97105-900, Santa

Maria, RS, Brasil. E-mail: cargnelutti@pq.cnpq.br. *Autor para correspondência.

"Departamento de Estatística, Universidade Federal do Rio Grande do Sul (UFRGS), Porto Alegre, RS, Brasil.

IIIFundação Estadual de Pesquisa Agropecuária (FEPAGRO), Porto Alegre, RS, Brasil. 
individual e conjunta dos dados experimentais resultantes de ensaios realizados em uma série de ambientes. Essas metodologias devem ser empregadas quando há interação GxA (CRUZ \& REGAZZI, 1997; CRUZ \& CARNEIRO, 2003) e podem ser agrupadas em classes.

Aquelas baseadas em análises de variância informam sobre a estabilidade dos genótipos avaliados. As estimativas do parâmetro de estabilidade são expressas em componentes quadráticos (quadrados médios ou componentes de variância) que, em certos casos, podem ser de baixa precisão (CRUZ, 2001). Entretanto, estes métodos proporcionam resultados de fácil interpretação e são vantajosos por serem aplicáveis mesmo quando o número de ambientes for relativamente reduzido. Entre essas metodologias, estão os métodos propostos por YATES \& COCHRAN (1938) (método tradicional), PLAISTED \& PETERSON (1959), WRICKE (1965) e ANNICCHIARICO(1992).

Em outra classe de métodos, a análise de adaptabilidade e estabilidade é realizada a partir de equações de regressão linear, em que a variável dependente, geralmente a produtividade de grãos ou frutos (ou seus componentes), é expressa em função de um índice ambiental que mede a qualidade dos ambientes avaliados (CRUZ, 2001). Nesta classe estão as metodologias propostas por FINLAY \& WILKINSON (1963), EBERHART \& RUSSELL(1966)e TAI (1971).

As metodologias de análise de adaptabilidade e estabilidade baseadas em regressão bissegmentada não-linear (TOLER, 1990) e linear (SILVA \& BARRETO, 1985; CRUZ et al., 1989) contemplam uma variável indexadora que permite avaliar o comportamento dos genótipos de forma diferenciada nos ambientes desfavoráveis (que apresentam valores negativos do índice ambiental) e favoráveis (que apresentam valores positivos do índice ambiental). Já as metodologias de HUEHN (1990) e LIN \& BINNS (1988) modificada por CARNEIRO (1998), inserem-se na classe de análise baseada em estatísticas nãoparamétricas.

A análise Additive Main effects and Multiplicative Interaction (AMMI), que significa análises de efeitos principais aditivos e interação multiplicativa, constitui outra classe. Esta metodologia combina a análise de variância dos efeitos aditivos principais de genótipos e ambientes com a análise de componentes principais do efeito multiplicativo da interação GxA (ZOBEL et al., 1988; DUARTE \& VENCOVSKY, 1999). Resultados preliminares confirmam a hipótese de que o primeiro componente principal da interação (IPCA $)$ é melhor que a análise de regressão linear na explicação da interação GxA.
O número de ambientes disponíveis, a precisão requerida e o tipo de informação desejada são critérios para a escolha do método que ofereça maior segurança na indicação de cultivares (CRUZ \& REGAZZI, 1997). Havendo concordância entre os métodos, em relação aos parâmetros de adaptabilidade e estabilidade, há possibilidade de escolher aquele de simples execução e de fácil interpretação. No entanto, caso haja discordância entre os métodos, a indicação de cultivares passa a depender do método utilizado, havendo necessidade de escolha do método mais eficiente.

Comparações entre metodologias de análise de adaptabilidade e estabilidade têm sido realizadas em trabalhos de SCAPIM et al. (2000), ROSSE et al. (2002), MURAKAMI et al. (2004), SABAGHNIA et al. (2006), SILVA\& DUARTE (2006), MOHAMMADI et al. (2007), SOUZA et al. (2007) e MOHAMMADI \& AMRI (2008). Além desses trabalhos, CARGNELUTTI FILHO et al. (2007) compararam metodologias baseadas em análise de variância, regressão linear e estatística não-paramétrica, considerando dados de produtividade de grãos de milho do Estado do Rio Grande do Sul. No entanto, comparações com metodologias baseadas em regressão bissegmentada linear e não-linear e análise AMMI são pouco conhecidas e carecem de pesquisas adicionais. Assim, o objetivo deste trabalho foi comparar métodos de análise de adaptabilidade e estabilidade em relação à produtividade de grãos, em ensaios de competição de cultivares de milho (Zea mays L.), conduzidos no Estado do Rio Grande do Sul, nos anos agrícolas 2002/ 2003, 2003/2004 e 2004/2005.

\section{MATERIAL E MÉTODOS}

Foram usados dados de produtividade de grãos, corrigidos a 13\% de umidade, de 65 ensaios de competição de cultivares de milho, realizados no Estado do Rio Grande do Sul. Os ensaios foram classificados em oito grupos de experimentos conforme a categoria (estadual e indicado), o ciclo (precoce e superprecoce) e o ano agrícola (2002/2003, 2003/2004 e 2004/2005) (Tabela 1). Em todos os ensaios, utilizou-se o delineamento blocos ao acaso com três repetições, sendo as unidades experimentais constituídas de duas fileiras com $5 \mathrm{~m}$ de comprimento, espaçadas em $0,8 \mathrm{~m}$.

Em relação à produtividade de grãos de milho, foram executadas as análises de variância para cada um dos 65 ensaios. Foram realizadas, em seguida, a análise de variância conjunta e o teste $\mathrm{F}$ a $1 \%$ de probabilidade de erro, para cada grupo de experimento. Depois, foram realizadas as análises de adaptabilidade e estabilidade, pelos métodos baseados em análise de variância: YATES \& COCHRAN (1938) ou tradicional 
Tabela 1 - Análise de variância conjunta e significância do quadrado médio (QM) das fontes de variação em relação à produtividade de grãos, em t ha ${ }^{-1}$, e média, coeficiente de variação experimental (CV) e relação entre o maior e o menor quadrado médio residual dos ambientes $\left(\mathrm{QMr}^{+} / \mathrm{QMr}^{-}\right)$de cada um dos oito grupos de experimentos de milho.

\begin{tabular}{|c|c|c|c|c|c|c|c|c|}
\hline \multirow[t]{2}{*}{ Causas de variação } & \multicolumn{2}{|c|}{$\begin{array}{l}\text { Estadual Superprecoce } \\
\text { 2002/2003 (ESP3) }\end{array}$} & \multicolumn{2}{|c|}{$\begin{array}{l}\text { Indicado Superprecoce } \\
\text { 2002/2003 (ISP3) }\end{array}$} & \multicolumn{2}{|c|}{$\begin{array}{l}\text { Estadual Superprecoce } \\
\text { 2003/2004 (ESP4) }\end{array}$} & \multicolumn{2}{|c|}{$\begin{array}{l}\text { Indicado Superprecoce } \\
\text { 2003/2004 (ISP4) }\end{array}$} \\
\hline & GL & QM & GL & QM & GL & QM & GL & QM \\
\hline Blocos/Ambientes & 18 & 1,9130 & 10 & 1,9736 & 24 & 6,0871 & 14 & 5,0411 \\
\hline Cultivares (C) & 10 & $31,7526 * *$ & 17 & $7,9738 * *$ & 8 & $18,0978 * *$ & 15 & $24,0217 * *$ \\
\hline Ambientes (A) & 8 & $146,9387 * *$ & 4 & $352,1732 * *$ & 11 & $135,1371 * *$ & 6 & $131,2104 * *$ \\
\hline Interação CxA & 80 & $1,9431 * *$ & 68 & $2,8517^{\mathrm{ns}}$ & 88 & $1,4096 * *$ & 90 & $2,3519 * *$ \\
\hline Resíduo & 180 & 0,8863 & 170 & 2,2173 & 192 & 0,8632 & 210 & 0,8031 \\
\hline Média & & 7,27 & & 7,76 & & 8,10 & & 8,40 \\
\hline CV (\%) & & 12,95 & & 19,20 & & 11,46 & & 10,66 \\
\hline \multirow[t]{3}{*}{$\mathrm{QMr}^{+} / \mathrm{QMr}^{-}$} & & 7,05 & & 5,41 & & 6,89 & & 4,94 \\
\hline & \multicolumn{2}{|c|}{$\begin{array}{l}\text { Estadual Precoce } \\
\text { 2004/2005 (EP5) }\end{array}$} & \multicolumn{2}{|c|}{$\begin{array}{l}\text { Estadual Superprecoce } \\
\text { 2004/2005 (ESP5) }\end{array}$} & \multicolumn{2}{|c|}{$\begin{array}{l}\text { Indicado Precoce } \\
\text { 2004/2005 (IP5) }\end{array}$} & \multicolumn{2}{|c|}{$\begin{array}{l}\text { Indicado Superprecoce } \\
2004 / 2005 \text { (ISP5) }\end{array}$} \\
\hline & GL & $\mathrm{QM}$ & GL & $\mathrm{QM}$ & GL & QM & GL & $\mathrm{QM}$ \\
\hline Blocos/Ambientes & 20 & 1,6609 & 22 & 1,5585 & 10 & 6,2042 & 12 & 3,8718 \\
\hline Cultivares (C) & 31 & $11,8743 * *$ & 16 & $12,8977 * *$ & 29 & $6,5034 * *$ & 11 & $11,7032 * *$ \\
\hline Ambientes (A) & 9 & $65,8625^{* *}$ & 10 & $28,7338 * *$ & 4 & $339,9175^{* *}$ & 5 & $189,1887 * *$ \\
\hline Interação CxA & 279 & $1,7411 * *$ & 160 & $1,4832 * *$ & 116 & $1,7627 * *$ & 55 & $1,2142 * *$ \\
\hline Resíduo & 620 & 0,5909 & 352 & 0,4178 & 290 & 0,7458 & 132 & 0,4364 \\
\hline Média & & 4,46 & & 4,68 & & 5,61 & & 4,96 \\
\hline CV $(\%)$ & & 17,24 & & 13,82 & & 15,38 & & 13,33 \\
\hline $\mathrm{QMr}^{+} / \mathrm{QMr}^{-}$ & & 3,57 & & 2,28 & & 7,34 & & 2,16 \\
\hline
\end{tabular}

(1) Na categoria dos ensaios estaduais, as cultivares avaliadas foram aquelas ainda não indicadas aos produtores e as indicadas foram estudadas na categoria dos ensaios indicados. $* *$ Significativo a $1 \%$ de probabilidade pelo teste $\mathrm{F}$. ${ }^{\text {ns }}$ Não-significativo.

(TR), PLAISTED \& PETERSON (1959)(PP), WRICKE (1965) (WR) e ANNICCHIARICO (1992) (AN); em regressão linear: FINLAY \& WILKINSON (1963)(FW), EBERHART \& RUSSELL (1966)(ER)e TAI(1971)(TA); em regressão bissegmentada não-linear: TOLER (1990) (TO); em regressão bissegmentada linear: SILVA \& BARRETO (1985) (SB) e CRUZ et al. (1989) (CR); em estatísticas não-paramétricas: HUEHN (1990) (HU) e LIN \& BINNS (1988) modificado por CARNEIRO (1998) (LB) e análise AMMI (ZOBEL et al., 1988) (AM).

Assim, foram obtidas para cada cultivar, em cada grupo de experimentos, a média de produtividade de grãos e as estimativas dos parâmetros de estabilidade e/ou adaptabilidade para a indicação de cultivares em ambientes gerais, desfavoráveis e favoráveis, em relação aos métodos: TR - parâmetro de estabilidade expresso pela variação de ambientes dentro de cada genótipo $\left(\mathrm{TRQM}_{\mathrm{AG}}\right)$; $\mathrm{PP}$ - parâmetro de estabilidade obtido pela média aritmética dos componentes de variância da interação entre pares de genótipos x ambientes $\left(\mathrm{PPW}_{\mathrm{i}}\right)$; WR - parâmetro de estabilidade estimado pela decomposição da soma de quadrados de genótipos $\mathrm{x}$ ambientes nas partes devidas a genótipos isolados $\left(\mathrm{WRW}_{\mathrm{i}}\right)$; $\mathrm{AN}$ - parâmetros de estabilidade, medidos pela superioridade do genótipo em relação à média de cada ambiente, obtendo-se um índice de indicação de cultivares em ambientes gerais $\left(\mathrm{ANW}_{\mathrm{ig}}\right)$, grupo de ambientes desfavoráveis $\left(\mathrm{ANW}_{\mathrm{id}}\right)$ e grupo de ambientes favoráveis $\left(\mathrm{ANW}_{\mathrm{if}}\right)$; $\mathrm{FW}^{\mathrm{id}}$ coeficiente de regressão $(\mathrm{FWb})$ como parâmetro de adaptabilidade e estabilidade; ER - coeficiente de regressão (ERb) como parâmetro de adaptabilidade e coeficiente de determinação $\left(E^{2} R^{2}\right)$ como medida de estabilidade; TA - o parâmetro TAb avalia a adaptabilidade e o TA $\lambda$, a estabilidade; TO coeficientes de regressão como parâmetro de adaptabilidade em ambientes desfavoráveis ( $\mathrm{TOb}$ ) e favoráveis $\left(\mathrm{TOb}_{2 \mathrm{i}}\right)$; $\mathrm{SB}$ - coeficientes de regressão colmo parâmetro de adaptabilidade em ambientes desfavoráveis $\left(\mathrm{SBb}_{13}\right)$ e favoráveis $\left(\mathrm{SBb}_{12}\right)$ e coeficiente de determinação $\left(\mathrm{SBR}^{2}\right)$ como medida de estabilidade; CR - coeficientes de regressão como parâmetro de adaptabilidade em ambientes desfavoráveis $\left(\mathrm{CRb}_{1 \mathrm{i}}\right) \mathrm{e}$ favoráveis $\left(\mathrm{CRb}_{12 \mathrm{i}}\right)$ e coeficiente de determinação 
$\left(\mathrm{CRR}^{2}\right)$ como medida de estabilidade; $\mathrm{HU}$ - estimativas dos parâmetros de estabilidade baseadas na classsificação das cultivares nos ambientes em relação às médias originais das cultivares nos vários ambientes (HUS, $\mathrm{HUS}_{2}$ e $\mathrm{HUS}_{3}$ ); LB - estimativas dos parâmetros de estabilidade para indicação de cultivares em ambientes gerais $\left(\mathrm{LBP}_{\mathrm{ig}}\right)$, grupo de ambientes desfavoráveis $\left(\mathrm{LBP}_{\text {id }}\right)$ e grupo de ambientes favoráveis $\left(\mathrm{LBP}_{\mathrm{if}}\right)$; e AM - parâmetro de estabilidade medido pela magnitude dos escores (valor absoluto) do primeiro componente principal de interação GxA (IPCA).

Para verificar o grau de associação entre a estimativa da média e dos parâmetros de adaptabilidade e estabilidade dos diferentes métodos (Média, TRQM PPW $, \mathrm{WRW}_{\mathrm{i}}, \mathrm{ANW}_{\mathrm{iq}}, \mathrm{ANW}_{\mathrm{id}}, \mathrm{ANW}_{\mathrm{if}}, \mathrm{FWb}, \mathrm{ERb}, \mathrm{ERR}^{2}$, $\mathrm{TAb},{ }^{\mathrm{i}} \mathrm{TA} \lambda, \mathrm{TOb}_{1 \mathrm{i}}, \mathrm{T}^{\mathrm{ig}} \mathrm{Ob}_{2 \mathrm{i}}, \mathrm{SBb}_{1 \mathrm{id}}, \mathrm{SBb}_{12 \mathrm{i}}, \mathrm{SBR}^{2}, \mathrm{CRb}_{1 \mathrm{i}}$, $\mathrm{CRb}_{12 \mathrm{i}}, \mathrm{CRR}^{2}, \mathrm{HUS}_{1}, \mathrm{HUS}_{2}, \mathrm{HUS}_{3}, \mathrm{LBP}_{\mathrm{ig}}, \mathrm{LBP}_{\mathrm{id}}, \mathrm{LBP}_{\text {if }}{ }_{\mathrm{if}} \mathrm{e}$ IPCA ${ }_{1}$ ), em cada grupo de experimentos, foi utilizado o coeficiente de correlação por postos de Spearman ( $\mathrm{r}$ ) entre os 351 pares de estimativas (combinação das 27 estimativas duas a duas). Assim, foram obtidas oito matrizes (oito grupos de experimentos) de dimensão 27. Em seguida, foi obtida a matriz média por meio do cálculo da média entre os $r_{\mathrm{s}}$ das oito matrizes.

Afim de verificar se as inferências poderiam ser realizadas com a matriz média dos $r$, foi calculado o coeficiente de correlação de Pearson ( $\mathrm{r}$ ) entre cada par de matrizes (oito matrizes e a matriz média) e sua significância foi verificada pelo teste $\mathrm{t}$ de Student, a $1 \%$ de probabilidade de erro, com 349 graus de liberdade. Quanto mais próximo de um for a estimativa obtida, maior concordância há entre a matriz do grupo de experimento e a matriz média.

A análise AMMI foi realizada com a rotina do programa SAS apresentada em DUARTE \& VENCOVSKY (1999) e as demais análises foram realizadas com o uso dos programas computacionais GENES (CRUZ, 2001) e Estabilidade(UNIVERSIDADE FEDERAL DE LAVRAS, 2000), além do aplicativo Office Excel.

\section{RESULTADOS E DISCUSSÃO}

A relação entre o maior e o menor quadrado médio residual dos ambientes $\left(\mathrm{QMr}^{+} / \mathrm{QMr}^{-}\right)$oscilou entre 2,16 (grupo indicado superprecoce 2004/2005) e 7,34 (grupo indicado precoce 2004/2005) (Tabela 1). Os testes estatísticos de Bartlett e F máximo, entre outros, são indicados para verificar a homogeneidade das variâncias residuais. Entretanto, GOMES (1990) e CRUZ \& REGAZZI (1997) consideram as variâncias residuais homogêneas e a análise conjunta adequada quando a relação $\mathrm{QMr}^{+} / \mathrm{QMr}$ é menor que sete. Pela proximidade a esse valor, os grupos de experimentos estadual superprecoce 2002/2003 $\left(\mathrm{QMr}^{+} / \mathrm{QMr}=7,05\right)$ e indicado precoce $2004 / 2005\left(\mathrm{QMr}^{+} / \mathrm{QMr}^{-}=7,34\right)$ também foram considerados homogêneos quanto às variâncias residuais, o que possibilita a realização da análise conjunta.

A análise de variância conjunta, em relação à produtividade de grãos, revelou presença de interação cultivar $\mathrm{x}$ ambiente $(\mathrm{CxA})(\mathrm{P} \leq 0,01)$ nos grupos de experimentos, com exceção do grupo indicado superprecoce 2002/2003 $(\mathrm{P}=0,09)$. No entanto, PERECIN \& CARGNELUTTI FILHO (2008) salientam que o uso de nível de significância menos rigoroso $(\mathrm{P}=0,25)$ para interpretação do efeito da interação "por experimento", em relação às demais fontes de variação da análise de variância, pode captar efeitos importantes. Com base nesse critério se aceita que, no grupo indicado superprecoce 2002/2003, há interação CxA. A interação revela comportamento diferenciado das cultivares nos ambientes e, assim, a indicação de cultivares, por meio da análise de adaptabilidade e estabilidade, é um procedimento adequado (Tabela 1).

De maneira geral, os coeficientes de correlação de Spearman ( $\mathrm{r}_{\mathrm{s}}$ ), para cada par de estimativas dos parâmetros, foram similares entre os oito grupos de experimentos, mostrando as mesmas tendências, diferindo um pouco quanto à magnitude das associações. Os coeficientes de correlação de Pearson (r) foram significativos e de magnitude no mínimo igual a $0,50(r \geq 0,50)$ entre os pares de matrizes, o que evidencia boa concordância entre os grupos de experimentos. Já entre a matriz de cada grupo de experimento e a matriz média dos oito grupos de experimentos houve associação linear significativa e de alta magnitude $(r \geq 0,80)$ (Tabela 2$)$, o que demonstra que as inferências, de um modo geral, podem ser realizadas em nível de média dos oito grupos (Tabela 3 ).

As inferências em relação aos métodos YATES \& COCHRAN (1938) ou tradicional (TR), PLAISTED \& PETERSON (1959)(PP), WRICKE(1965) (WR), ANNICCHIARICO (1992) (AN), EBERHART \& RUSSELL(1966)(ER), TAI(1971)(TA) e LIN \& BINNS (1988) modificado por CARNEIRO (1998) (LB) comparados no trabalho de CARGNELUTTI FILHO et al. (2007), com base em quatro grupos de experimentos de milho, estão de acordo com as inferências obtidas neste trabalho, considerando-se outros oito grupos de experimentos, o que revela consistência das conclusões. Assim, neste trabalho, são enfatizados, na discusssão dos resultados, os demais métodos (FINLAY \& WILKINSON (1963) (FW), TOLER (1990) (TO), SILVA\& BARRETO(1985)(SB), CRUZ etal.(1989) (CR), HUEHN (1990) (HU) e análise AMMI (ZOBEL et al., 1988) (AM)).

O coeficiente de correlação de Spearman $\left(\mathrm{r}_{\mathrm{s}}\right)$ foi positivo e de alta magnitude entre a média e as estimativas de estabilidade do método de AN para os 
Tabela 2 - Coeficiente de correlação de Pearson (r) entre cada par de matrizes (oito matrizes e a matriz média) de correlação de Spearman entre a média e as estimativas dos parâmetros de adaptabilidade e/ou estabilidade, obtidos por 13 métodos, em relação à produtividade de grãos de cultivares de milho.

\begin{tabular}{|c|c|c|c|c|c|c|c|c|}
\hline Grupo ${ }^{(1)}$ & ISP3 & ESP4 & ISP4 & EP5 & ESP5 & IP5 & ISP5 & Média \\
\hline ESP3 & $0,58 * *$ & $0,71 * *$ & $0,69 * *$ & $0,68 * *$ & $0,69 * *$ & $0,68 * *$ & $0,80 * *$ & $0,87 * *$ \\
\hline ISP3 & & $0,50 * *$ & $0,58 * *$ & $0,70 * *$ & $0,64 * *$ & $0,80 * *$ & $0,69 * *$ & $0,80 * *$ \\
\hline ESP4 & & & $0,58 * *$ & $0,81 * *$ & $0,59 * *$ & $0,58 * *$ & $0,68 * *$ & $0,82 * *$ \\
\hline ISP4 & & & & $0,58^{* *}$ & $0,83 * *$ & $0,56^{* *}$ & $0,76^{* *}$ & $0,83^{* *}$ \\
\hline EP5 & & & & & $0,61 * *$ & $0,77 * *$ & $0,66^{* *}$ & $0,86^{* *}$ \\
\hline ESP5 & & & & & & $0,67^{* *}$ & $0,79 * *$ & $0,86^{* *}$ \\
\hline IP5 & & & & & & & $0,75^{* *}$ & $0,85^{* *}$ \\
\hline ISP5 & & & & & & & & $0,90 * *$ \\
\hline
\end{tabular}

(1) Descrição dos grupos de experimentos na Tabela 1. ** Significativo a $1 \%$ de probabilidade pelo teste $\mathrm{t}$ de Student, com 349 graus de liberdade.

ambientes gerais $\left(\mathrm{ANW}_{\mathrm{ig}}\right)$, desfavoráveis $\left(\mathrm{ANW}_{\mathrm{id}}\right) \mathrm{e}$ favoráveis $\left(\mathrm{ANW}_{\mathrm{if}}\right)\left(\right.$ média $\mathrm{x} \mathrm{ANW}_{\mathrm{ig}}=0,97$, média $\mathrm{x}$

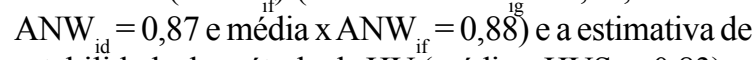
estabilidade do método de $\mathrm{HU}^{\text {if }}$ (média $\mathrm{HUS}_{3}=0,83$ ), e negativo, e de alta magnitude $\left(\mathrm{r}_{\mathrm{s}} \leq-0,86\right) \mathrm{com}$ as estimativas de estabilidade do método de LB para os ambientes gerais $\left(\mathrm{LBP}_{\mathrm{ig}}\right)$, desfavoráveis $\left(\mathrm{LBP}_{\mathrm{id}}\right)$ e favoráveis $\left(\mathrm{LBP}_{\mathrm{if}}\right)$. Assim, as cultivares mais indicadas por AN (maiores escores) e por LB (menores escores) são as mais produtivas, o que demonstra concordância entre esses dois métodos. Reforçam essa afirmação as correlações negativas e de alta magnitude $\left(r_{s} \leq-0,64\right)$ entre as estatísticas $\mathrm{ANW}_{\mathrm{ig}}$, $\mathrm{ANW}_{\text {if }}$ e $\mathrm{ANW}_{\mathrm{id}}$ com as $\mathrm{LBP}_{\mathrm{ig}}, \mathrm{LBP}_{\mathrm{id}}$ e LBP (Tabela 3 ). Resultados semelhantes foram constatados em SILVA \& DUARTE (2006) e CARGNELUTTI FILHO et al. (2007). Portanto, o uso desses métodos resulta em informações redundantes. Por outro lado, as estimativas dos demais métodos não evidenciaram associação com a média, o que mostra que não necessariamente as mais produtivas apresentam melhor adaptabilidade e estabilidade e viceversa.

A inexistência de interação CxA possibilita indicar cultivares para todos os ambientes com base apenas na média. Assim, a alta concordância das estatísticas $A N W_{i \mathrm{~g}}, \mathrm{ANW}_{\mathrm{if}}, \mathrm{ANW}_{\mathrm{id}}, \mathrm{LBP}_{\mathrm{ig}}, \mathrm{LBP}_{\mathrm{id}}$ e $\mathrm{LBP}_{\mathrm{if}}$ com a média de produtividade de grãos sugere possível ineficiência desses métodos, haja vista que a indicação de cultivares com base nas estatísticas $\mathrm{ANW}_{\mathrm{ig}}, \mathrm{ANW}_{\mathrm{if}}$, $\mathrm{ANW}_{\mathrm{id}}, \mathrm{LBP}_{\mathrm{ig}}, \mathrm{LBP}_{\mathrm{id}}$ e LBP if ou na média é praticamente a mesma. Assim, os efeitos da interação CxA não estariam sendo considerados.

Houve concordância perfeita $\left(r_{s}=1\right)$ entre as estimativas dos parâmetros de estabilidade obtidas pelos método PP $\left(\mathrm{PPW}_{\mathrm{i}}\right)$ e WR $\left(\mathrm{WRW}_{\mathrm{i}}\right)$, mostrando que a indicação de cultivares é a mesma, independentemente do método, e que o uso de ambos é desnecessário. Resultados estão de acordo com OLIVEIRA(1976), citado por CRUZ\& REGAZZI(1997), SILVA\& DUARTE (2006) e CARGNELUTTI FILHO et al. (2007), que obtiveram $r_{s}$ iguais a $0,9857,1$ e 1 , respectivamente. As estimativas dos parâmetros desses dois métodos, quando comparadas com as estimativas dos demais métodos, mostraram-se concordantes em relação aos coeficientes de determinação $\left(\mathrm{ERR}^{2}\right)$ do método de $\mathrm{ER}\left(\mathrm{r}_{\mathrm{s}}=-0,72\right),\left(\mathrm{SBR}^{2}\right)$ do método de $\mathrm{SB}\left(\mathrm{r}_{\mathrm{s}}=-0,61\right)$ e $\left(\mathrm{CRR}^{2}\right)$ do método de $\mathrm{CR}$ $\left(r_{s}=-0,62\right)$, com o parâmetro de estabilidade do método de TA $($ TA $\lambda)\left(r_{\mathrm{s}}=0,83\right)$, com os parâmetros de estabilidade $\left(\mathrm{HUS}_{1}^{\mathrm{s}}, \mathrm{r}_{\mathrm{s}}=0,50 ; \mathrm{HUS}_{2}, \mathrm{r}_{\mathrm{s}}=0,54\right)$ do método de HU e ainda com o parâmetro de estabilidade da AM $\left(\right.$ IPCA $\left._{1}\right)\left(r_{s}=0,61\right)$, revelando as mesmas informações (Tabela 3). Portanto, deve-se interpretar que cultivares mais indicadas (menores escores), ou seja, mais estáveis em relação aos parâmetros $\mathrm{PPW}_{\mathrm{i}}$ e $\mathrm{WRW}_{\mathrm{i}}$, estão associadas a maiores escores de $\mathrm{ERR}^{2}, \mathrm{SBR}^{2} \mathrm{e}$ $\mathrm{CRR}^{2}$ (mais estáveis) e menores escores de TA $\lambda$, HUS $\mathrm{HUS}_{2}$ e IPCA 1 (mais estáveis), o que revela concordância nas indicações de cultivares quanto a esses parâmetros. SILVA \& DUARTE (2006) também obtiveram associação linear de magnitude semelhante dos dois métodos (PP e WR) com análise AMMI ( $\mathrm{r}_{\mathrm{s}}=$ 0,72 ) e com a média dos postos dos parâmetros HUS $\mathrm{HUS}_{2}$ e $\mathrm{HUS}_{3}$ do método de $\mathrm{HU}\left(\mathrm{r}_{\mathrm{s}}=0,79\right)$.

Houve alta concordância entre a estimativa do parâmetro de estabilidade do método TR $\left(\mathrm{TRQM}_{\mathrm{A} / \mathrm{G}}\right)$ e as estimativas dos parâmetros de adaptabilidade dos métodos de $\mathrm{ER}(\mathrm{ERb})\left(\mathrm{r}_{\mathrm{s}}=0,92\right), \mathrm{TA}(\mathrm{TAb})\left(\mathrm{r}_{\mathrm{s}}=0,92\right) \mathrm{e}$ $\mathrm{CR}\left(\mathrm{CRb}_{1 \mathrm{i}}\right)\left(\mathrm{r}_{\mathrm{s}}=0,86\right)$. Cultivares com valores de $\mathrm{ERb}$, $\mathrm{TAb}$ e $\mathrm{CRb}{ }_{1 \mathrm{i}}{ }^{\mathrm{s}}$ menores que um, de modo geral, são indicadas para ambientes desfavoráveis. Então, esses resultados reforçam a afirmação de que as cultivares mais indicadas pelo método TR (menores escores), ou seja, mais estáveis, possivelmente, são as mais 
Tabela 3 - Matriz de coeficiente de correlação de Spearman (média dos coeficientes de oito grupos de experimentos ${ }^{(1)}$ ) entre a média e as estimativas dos parâmetros de adaptabilidade e/ou estabilidade, obtidos por 13 métodos, em relação à produtividade de grãos de cultivares de milho.

\begin{tabular}{|c|c|c|c|c|c|c|c|c|c|c|c|c|c|}
\hline Parâmetro (2) & $\begin{array}{c}\text { TRQM } \\
\mathrm{A} / \mathrm{G}\end{array}$ & $\mathrm{PPW}_{\mathrm{i}}$ & $\mathrm{WRW}_{\mathrm{i}}$ & $\mathrm{ANW}_{\text {ig }}$ & $\mathrm{ANW}_{\text {id }}$ & $\mathrm{ANW}_{\text {if }}$ & $\mathrm{FWb}$ & $\mathrm{ERb}$ & $\mathrm{ERR}^{2}$ & $\mathrm{TAb}$ & $\mathrm{TA} \lambda$ & $\mathrm{TOb}_{1 \mathrm{i}}$ & $\mathrm{TOb}_{2 \mathrm{i}}$ \\
\hline Média & 0,32 & 0,09 & 0,09 & 0,97 & 0,87 & 0,88 & $-0,26$ & 0,28 & 0,03 & 0,28 & 0,08 & 0,28 & 0,10 \\
\hline $\mathrm{TRQM}_{\mathrm{A} / \mathrm{G}}$ & & 0,26 & 0,26 & 0,20 & 0,02 & 0,52 & 0,58 & 0,92 & 0,23 & 0,92 & 0,26 & 0,36 & 0,50 \\
\hline $\mathrm{PPW}_{\mathrm{i}}$ & & & 1,00 & 0,02 & 0,03 & 0,07 & $-0,07$ & 0,02 & $-0,72$ & 0,02 & 0,83 & 0,04 & 0,05 \\
\hline $\mathrm{WRW}_{\mathrm{i}}$ & & & & 0,02 & 0,03 & 0,07 & $-0,07$ & 0,02 & $-0,72$ & 0,02 & 0,83 & 0,04 & 0,05 \\
\hline $\mathrm{ANW}_{\text {ig }}$ & & & & & 0,92 & 0,85 & $-0,37$ & 0,18 & 0,06 & 0,18 & $-0,02$ & 0,20 & 0,07 \\
\hline $\mathrm{ANW}_{\text {id }}$ & & & & & & 0,66 & $-0,53$ & $-0,01$ & $-0,06$ & $-0,01$ & $-0,03$ & 0,10 & $-0,03$ \\
\hline $\mathrm{ANW}_{\text {if }}$ & & & & & & & $-0,04$ & 0,50 & 0,20 & 0,50 & 0,03 & 0,39 & 0,18 \\
\hline $\mathrm{FWb}$ & & & & & & & & 0,69 & 0,43 & 0,69 & $-0,02$ & 0,31 & 0,23 \\
\hline $\mathrm{ERb}$ & & & & & & & & & 0,48 & 1,00 & 0,01 & 0,36 & 0,52 \\
\hline $\mathrm{ERR}^{2}$ & & & & & & & & & & 0,48 & $-0,77$ & 0,12 & 0,25 \\
\hline $\mathrm{TAb}$ & & & & & & & & & & & 0,01 & 0,36 & 0,52 \\
\hline $\mathrm{TA} \lambda$ & & & & & & & & & & & & 0,07 & 0,02 \\
\hline $\mathrm{TOb}_{1 \mathrm{i}}$ & & & & & & & & & & & & & $-0,46$ \\
\hline & $\mathrm{SBb}_{1 \mathrm{i}}$ & $\mathrm{SBb}_{12 \mathrm{i}}$ & $\mathrm{SBR}^{2}$ & $\mathrm{CRb}_{1 \mathrm{i}}$ & $\mathrm{CRb}_{12 \mathrm{i}}$ & $\mathrm{CRR}^{2}$ & $\mathrm{HUS}_{1}$ & $\mathrm{HUS}_{2}$ & $\mathrm{HUS}_{3}$ & $\mathrm{LBP}_{\text {ig }}$ & $\mathrm{LBP}_{\text {id }}$ & $\mathrm{LBP}_{\text {if }}$ & $\mathrm{IPCA}_{1}$ \\
\hline Média & 0,30 & 0,08 & 0,08 & 0,28 & 0,09 & 0,10 & 0,06 & 0,06 & 0,83 & $-0,98$ & $-0,86$ & $-0,88$ & 0,04 \\
\hline $\mathrm{TRQM}_{\mathrm{A} / \mathrm{G}}$ & 0,39 & 0,49 & 0,25 & 0,86 & 0,27 & 0,17 & 0,10 & 0,10 & 0,32 & $-0,28$ & $-0,04$ & $-0,54$ & 0,12 \\
\hline $\mathrm{PPW}_{\mathrm{i}}$ & 0,05 & 0,06 & $-0,61$ & 0,05 & 0,05 & $-0,62$ & 0,50 & 0,54 & 0,42 & 0,00 & $-0,02$ & $-0,02$ & 0,61 \\
\hline $\mathrm{WRW}_{\mathrm{i}}$ & 0,05 & 0,06 & $-0,61$ & 0,05 & 0,05 & $-0,62$ & 0,50 & 0,54 & 0,42 & 0,00 & $-0,02$ & $-0,02$ & 0,61 \\
\hline $\mathrm{ANW}_{\mathrm{ig}}$ & 0,22 & 0,06 & 0,09 & 0,18 & 0,05 & 0,12 & $-0,01$ & 0,00 & 0,79 & $-0,96$ & $-0,89$ & $-0,84$ & 0,02 \\
\hline $\mathrm{ANW}_{\text {id }}$ & 0,12 & $-0,04$ & $-0,04$ & $-0,04$ & 0,11 & 0,01 & $-0,02$ & $-0,02$ & 0,72 & $-0,88$ & $-0,97$ & $-0,66$ & 0,07 \\
\hline $\mathrm{ANW}_{\text {if }}$ & 0,42 & 0,16 & 0,21 & 0,56 & 0,01 & 0,17 & 0,03 & 0,03 & 0,74 & $-0,86$ & $-0,64$ & $-0,97$ & 0,01 \\
\hline $\mathrm{FWb}$ & 0,32 & 0,24 & 0,42 & 0,65 & 0,21 & 0,34 & $-0,01$ & $-0,02$ & $-0,27$ & 0,27 & 0,47 & 0,01 & $-0,09$ \\
\hline $\mathrm{ERb}$ & 0,39 & 0,51 & 0,48 & 0,90 & 0,32 & 0,39 & $-0,05$ & $-0,04$ & 0,19 & $-0,28$ & $-0,02$ & $-0,55$ & $-0,03$ \\
\hline $\mathrm{ERR}^{2}$ & 0,14 & 0,24 & 0,90 & 0,42 & 0,15 & 0,83 & $-0,42$ & $-0,44$ & $-0,28$ & $-0,10$ & 0,05 & $-0,24$ & $-0,40$ \\
\hline $\mathrm{TAb}$ & 0,39 & 0,51 & 0,48 & 0,90 & 0,32 & 0,39 & $-0,05$ & $-0,04$ & 0,19 & $-0,28$ & $-0,02$ & $-0,55$ & $-0,03$ \\
\hline $\mathrm{TA} \lambda$ & 0,07 & 0,04 & $-0,69$ & 0,04 & $-0,01$ & $-0,63$ & 0,52 & 0,53 & 0,38 & 0,01 & 0,04 & 0,00 & 0,43 \\
\hline \multirow[t]{2}{*}{$\mathrm{TOb}_{1 \mathrm{i}}$} & 0,98 & $-0,47$ & 0,17 & 0,54 & $-0,32$ & 0,08 & 0,07 & 0,04 & 0,26 & $-0,25$ & $-0,20$ & $-0,34$ & $-0,02$ \\
\hline & $\mathrm{SBb}_{1 \mathrm{i}}$ & $\mathrm{SBb}_{12 \mathrm{i}}$ & $\mathrm{SBR}^{2}$ & $\mathrm{CRb}_{1 \mathrm{i}}$ & $\mathrm{CRb}_{12 \mathrm{i}}$ & $\mathrm{CRR}^{2}$ & $\mathrm{HUS}_{1}$ & $\mathrm{HUS}_{2}$ & $\mathrm{HUS}_{3}$ & $\mathrm{LBP}_{\mathrm{ig}}$ & $\mathrm{LBP}_{\text {id }}$ & $\mathrm{LBP}_{\text {if }}$ & $\mathrm{IPCA}_{1}$ \\
\hline $\mathrm{TOb}_{2 \mathrm{i}}$ & $-0,43$ & 0,99 & 0,24 & 0,33 & 0,54 & 0,22 & $-0,11$ & $-0,08$ & 0,04 & $-0,10$ & 0,09 & $-0,26$ & 0,01 \\
\hline $\mathrm{SBb}_{1 \mathrm{i}}$ & & $-0,45$ & 0,19 & 0,57 & $-0,30$ & 0,11 & 0,07 & 0,04 & 0,27 & $-0,27$ & $-0,22$ & $-0,37$ & $-0,01$ \\
\hline $\mathrm{SBb}_{12 \mathrm{i}}$ & & & 0,22 & 0,32 & 0,57 & 0,21 & $-0,09$ & $-0,06$ & 0,04 & $-0,09$ & 0,10 & $-0,23$ & 0,03 \\
\hline $\mathrm{SBR}^{2}$ & & & & 0,43 & 0,11 & 0,85 & $-0,39$ & $-0,40$ & $-0,22$ & $-0,13$ & 0,03 & $-0,24$ & $-0,32$ \\
\hline $\mathrm{CRb}_{1 \mathrm{i}}$ & & & & & 0,04 & 0,33 & 0,00 & 0,02 & 0,22 & $-0,26$ & 0,01 & $-0,56$ & $-0,02$ \\
\hline $\mathrm{CRb}_{12 \mathrm{i}}$ & & & & & & 0,16 & $-0,13$ & $-0,09$ & 0,03 & $-0,12$ & $-0,11$ & $-0,11$ & 0,12 \\
\hline $\mathrm{CRR}^{2}$ & & & & & & & $-0,34$ & $-0,35$ & $-0,19$ & $-0,16$ & $-0,03$ & $-0,22$ & $-0,33$ \\
\hline $\mathrm{HUS}_{1}$ & & & & & & & & 0,96 & 0,44 & 0,01 & 0,02 & 0,00 & 0,22 \\
\hline $\mathrm{HUS}_{2}$ & & & & & & & & & 0,45 & 0,01 & 0,02 & 0,00 & 0,28 \\
\hline $\mathrm{HUS}_{3}$ & & & & & & & & & & $-0,77$ & $-0,72$ & $-0,71$ & 0,25 \\
\hline $\mathrm{LBP}_{\text {ig }}$ & & & & & & & & & & & 0,88 & 0,88 & 0,02 \\
\hline $\mathrm{LBP}_{\mathrm{id}}$ & & & & & & & & & & & & 0,65 & $-0,04$ \\
\hline $\mathrm{LBP}_{\text {if }}$ & & & & & & & & & & & & & 0,02 \\
\hline
\end{tabular}

(1) Descrição dos grupos de experimentos na Tabela 1.

(2) TRQM $_{\mathrm{A} / \mathrm{G}}$ : estabilidade do método tradicional; PPW $\mathrm{i}$ : estabilidade do método proposto por PLAISTED \& PETERSON (1959); WRW estabilidade do método proposto por WRICKE (1965); $\mathrm{ANW}_{\mathrm{ig}}, \mathrm{ANW}_{\mathrm{id}}$ e $\mathrm{ANW}_{\mathrm{if}}$ : estabilidade em ambientes gerais, grupo de ambientes desfavoráveis e grupo de ambientes favoráveis, respectivamente, conforme metodologia proposta por ANNICCHIARICO (1992); FWb: adaptabilidade do método de FINLAY \& WILKINSON (1963); ERb e ERR ${ }^{2}$ : adaptabilidade e estabilidade do método de EBERHART \& RUSSELL (1966); TAb e TA $\lambda$ : adaptabilidade e estabilidade do método de TAI (1971); TOb $_{1 \mathrm{i}}$ e TOb $_{2 \mathrm{i}}$ adaptabilidade, respectivamente, em ambientes desfavoráveis e favoráveis pelo método de TOLER (1990); $\mathrm{SBb}_{1 \mathrm{i}}, \mathrm{SBb}_{12 \mathrm{i}}$ e SBR${ }^{2}$ : adaptabilidade em ambientes desfavoráveis e

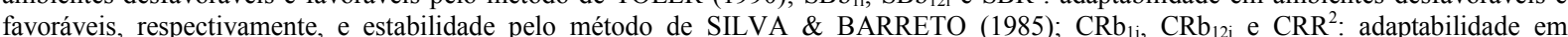
ambientes desfavoráveis e favoráveis, respectivamente, e estabilidade pelo método de CRUZ et al. (1989); HUS , HUS $_{2}$ e HUS H $_{3}$ estabilidade conforme HUEHN (1990); $\mathrm{LBP}_{\mathrm{ig}}, \mathrm{LBP}_{\text {if }}$ e $\mathrm{LBP}_{\mathrm{id}}$ : estabilidade em ambientes gerais, grupo de ambientes favoráveis e grupo de ambientes

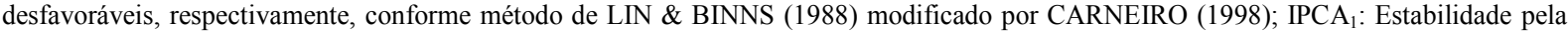
análise AMMI (ZOBEL et al., 1988).

adaptadas a ambientes desfavoráveis (CARGNELUTTI FILHO et al., 2007). Já SILVA \& DUARTE (2006) encontraram concordância do método TR com os métodos de FW $\left(r_{\mathrm{s}}=0,89\right), \mathrm{ER}\left(\mathrm{r}_{\mathrm{s}}=0,83\right)$ e TO $\left(\mathrm{r}_{\mathrm{s}}=0,86\right)$ e discordância com $\operatorname{LB}\left(r_{s}=-0,72\right)$. Em relação aos métodos com mais de um parâmetro, ressalta-se que a metodologia utilizada por SILVA \& DUARTE (2006) difere da utilizada neste trabalho. 
Entre os métodos que utilizam regressão (FW, ER, TA, TO, SB e CR), houve, de maneira geral, boa concordância entre as estimativas dos parâmetros de adaptabilidade ( $\mathrm{FWb}, \mathrm{ERb}, \mathrm{TAb}, \mathrm{TOb}_{1 \mathrm{i}}, \mathrm{TOb}_{2 \mathrm{i}}, \mathrm{SBb}_{1 \mathrm{i}}$, $\mathrm{SBb}_{12 \mathrm{i}}, \mathrm{CRb}_{1 \mathrm{i}}$ e $\mathrm{CRb}_{12 \mathrm{i}}$ ) e alta concordância entre as estimativas dos parâmetros de estabilidade (ERR2 x $\mathrm{TA} \lambda=-0,77 ; \mathrm{ERR}^{2} \times \mathrm{SBR}^{2}=0,90 ; \mathrm{ERR}^{2} \times \mathrm{CRR}^{2}=0,83$; $\mathrm{TA} \lambda \times \mathrm{SBR}^{2}=-0,69$; $\mathrm{TA} \lambda \times \mathrm{CRR}^{2}=-0,63 \mathrm{eSBR}^{2} \times \mathrm{CRR}^{2}=$ $0,85)$. A forte associação entre os métodos de TO e SB em relação às estimativas do parâmetro de adaptabilidade em ambientes desfavoráveis $\left(\mathrm{TOb}_{1 \mathrm{i}} \mathrm{x}\right.$ $\left.\mathrm{SBb}_{1 \mathrm{i}}=0,98\right)$ e favoráveis $\left(\mathrm{TOb}_{2 \mathrm{i}} \times \mathrm{SBb}_{12 \mathrm{i}}=0,99\right)$ comprova informações de mesma natureza. Resultados redundantes são obtidos pelo uso concomitante dos métodos ER e TA, devido à alta magnitude das correlações $\operatorname{ERb} \times \mathrm{TAb}\left(\mathrm{r}_{\mathrm{s}}=1\right)$ e $\mathrm{ERR}^{2} \times \mathrm{TA} \lambda\left(\mathrm{r}_{\mathrm{s}}=-0,77\right)$. CARGNELUTTI FILHO et al. (2007) encontraram valores de $r_{s}=1$ e $r_{s}=-0,88$ entre as estimativas dos parâmetros $\stackrel{\mathrm{s}}{\mathrm{E}} \mathrm{Rb}$ x TA $\mathrm{s} b$ e $\mathrm{ERR}^{2}$ x TA $\lambda$, respectivamente. Portanto, esses resultados indicam certo nível de redundância entre os métodos que utilizam análise de regressão, como destacado em SILVA \& DUARTE (2006). Pode-se inferir ainda que a maior redundância ocorre na estimativa dos parâmetros de estabilidade.

Quanto aos métodos baseados em estatísticas não-paramétricas, as estimativas dos parâmetros de estabilidade de HU ( HUS $_{1}, \mathrm{HUS}_{2}$ ) e LB para indicação de cultivares em ambientes gerais ( $\left.\mathrm{LBP}_{\mathrm{ig}}\right)$, desfavoráveis $\left(\mathrm{LBP}_{\mathrm{id}}\right)$ e favoráveis ( $\mathrm{LBP}$ ) nẵo revelaram associação $\left(\mathrm{r}_{\mathrm{s}}=0,02\right)$. No entanto, $\mathrm{O}_{\mathrm{if}}^{\mathrm{if}} \mathrm{HUS}_{3}$ definido como a soma dos desvios absolutos de cada classificação em relação à média das classificações (CRUZ \& CARNEIRO, 2003) foi discordante de LBP

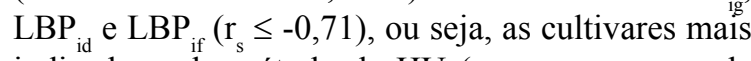
indicadas pelo método de HU (menores escores de $\mathrm{HUS}_{3}$ ) são as menos indicadas por LB (maiores escores de $\operatorname{LBP}_{\mathrm{ig}}, \mathrm{LBP}_{\mathrm{id}}$ e LBP if $_{\text {if }}$. SILVA \& DUARTE (2006) encontraram coeficiente de correlação de Spearman igual a $-0,03$ entre a média das ordens dos parâmetros de $\mathrm{HU}\left(\mathrm{HUS}_{1}, \mathrm{HUS}_{2}\right.$ e $\mathrm{HUS}_{3}$ ) e $\mathrm{LBP}_{\mathrm{ig}}$.

$\mathrm{A}$ análise AMMI revelou concordância $\left(r_{\mathrm{s}}=0,61\right)$ com os métodos PP e WR e ausência de associação com os métodos TR e AN. Em relação aos métodos que utilizam regressão, a análise AMMI mostrou concordância, em menor magnitude, com as estimativas dos parâmetros de estabilidade (IPCA $\mathrm{x}$ $\mathrm{ERR}^{2}=-0,40 ;$ IPCA $_{1} \times \mathrm{TA} \lambda=0,43 ; \mathrm{IPCA}_{1} \times \mathrm{SBR}^{2}=-0,32$; $\mathrm{IPCA}_{1} \times \mathrm{CRR}^{2}=-0,33$ ) e ausência de associação com as estimativas dos parâmetros de adaptabilidade (FWb, $\mathrm{ERb}, \mathrm{TAb}, \mathrm{TOb}_{1 \mathrm{i}}, \mathrm{TOb}_{2 \mathrm{i}}, \mathrm{SBb}_{1 \mathrm{i}}, \mathrm{SBb}_{2 \mathrm{i}}, \mathrm{CRb}_{1 \mathrm{i}}$ e CRb $\mathrm{CRi}_{12 \mathrm{i}}$ ). Esses resultados sugerem associação, apesar de fraca, entre os métodos quanto à estabilidade das cultivares. Concordância em pequena magnitude há entre o IPCA e a estimativa dos parâmetros de HU (HUS $-\mathrm{r}_{\mathrm{s}}=0,22$, $\mathrm{HUS}_{2}-\mathrm{r}_{\mathrm{s}}=0,28$ e $\mathrm{HUS}_{3}-\mathrm{r}_{\mathrm{s}}=0,25$ ) e ausência de associaçã̃o entre as estimativas dos parâmetros de estabilidade para indicação de cultivares em ambientes gerais $\left(\mathrm{LBP}_{\mathrm{ig}}\right)$, desfavoráveis $\left(\mathrm{LBP}_{\mathrm{id}}\right)$ e favoráveis $\left(\mathrm{LBP}_{\text {if }}\right)$ do método de LB.

Associação linear, de magnitude semelhante, da análise AMMI com os métodos PP e $\mathrm{WR}\left(\mathrm{r}_{\mathrm{s}}=0,72\right)$ e com ER $\left(\mathrm{r}_{\mathrm{s}}=0,52\right)$ foi obtida por SILVA \& DUARTE (2006). O sinal positivo da correlação $\left(r_{s}=0,52\right)$ é devido à estimativa do parâmetro utilizada para medir a estabilidade que foi o desvio da regressão $\left(\sigma^{2}{ }_{\mathrm{di}}\right)$, enquanto neste trabalho utilizou-se o coeficiente de determinação $\left(E^{2} R^{2}\right)$, que resultou em IPCA $x$ $\mathrm{ERR}^{2}=-0,40$. A interpretação correta é que menores desvios e maiores coeficientes de determinação indicam maior estabilidade, portanto, apesar da inversão de sinais, a informação dos trabalhos é concordante. Esses resultados corroboram SILVA \& DUARTE (2006), que salientam que esses métodos, de certa forma, se complementam, visto que o método de ER, em detrimento da análise AMMI, informa sobre a responsividade de cada genótipo em relação à melhoria ambiental. Por sua vez, a análise AMMI produz uma estimativa da contribuição genotípica, para a interação GxA, livre de ruídos, que o método de ER não possibilita.

De maneira geral, entre os 13 métodos comparados, o uso concomitante dos métodos PP e WR, AN e LB, ER e TA, e TO e SB é desnecessário. No entanto, o uso das informações de um deles aliadas às informações dos demais métodos de análise de adaptabilidade e estabilidade, considerando as particularidades de cada um, é adequada para uma melhor tomada de decisão em relação à indicação de cultivares.

\section{CONCLUSÕES}

Em relação à produtividade de grãos, há concordância na indicação de cultivares de milho pelos métodos de PLAISTED \& PETERSON (1959) e WRICKE (1965), de ANNICCHIARICO (1992) e LIN \& BINNS (1988) modificado por CARNEIRO (1998), de EBERHART \& RUSSELL (1966) e TAI (1971), e de TOLER (1990) e SILVA\& BARRETO (1985), o que torna desnecessário o uso concomitante desses métodos. Em menor grau de associação, a análise AMMI concorda com as estimativas dos parâmetros de estabilidade de PLAISTED \& PETERSON (1959), WRICKE (1965), EBERHART \& RUSSELL (1966), TAI (1971), SILVA\& BARRETO (1985) e CRUZ et al. (1989). Os métodos propostos por ANNICCHIARICO (1992) e por LIN \& BINNS (1988) modificado por CARNEIRO 
(1998) são associados com a média de produtividade de grãos.

\section{AGRADECIMENTOS}

Os autores agradecem aos pesquisadores que auxiliaram na realização dos experimentos, pela cessão dos dados. Ao Conselho Nacional de Desenvolvimento Científico e Tecnológico $(\mathrm{CNPq})$, pela concessão de bolsa de Produtividade em Pesquisa a Alberto Cargnelutti Filho e a Lindolfo Storck.

\section{REFERÊNCIAS}

ANNICCHIARICO, P. Cultivar adaptation and recomendation from alfafa trialsin Northern Italy. Journal of Genetics and Plant Breeding, v.4, p.269-278, 1992.

CARGNELUTTI FILHO, A. et al. Comparação de métodos de adaptabilidade e estabilidade relacionados à produtividade de grãos de cultivares de milho. Bragantia, v.66, p.571-578, 2007 .

CARNEIRO, P.C.S. Novas metodologias de análise da adaptabilidade e estabilidade de comportamento. 1998. 155f. Tese (Doutorado em Genética e Melhoramento) Universidade Federal de Viçosa.

CRUZ, C.D. Programa GENES - versão Windows. Aplicativo computacional em genética e estatística Viçosa: UFV, 2001. 648p.

CRUZ, C.D.; CARNEIRO, P.C.S. Modelos biométricos aplicados ao melhoramento genético. Viçosa: UFV, 2003 V.2.

CRUZ, C.D.; REGAZZI, A.J. Modelos biométricos aplicados ao melhoramento genético. 2.ed. Viçosa: UFV, 1997. 390p.

CRUZ, C.D. et al. An alternative approach to the stability analysis proposed by Silva e Barreto. Revista Brasileira de Genética, v.12, p.567-580, 1989.

DUARTE, J.B.; VENCOVSKY, R. Interação genótipos x ambientes: uma introdução à análise AMMI. Ribeirão Preto: Sociedade Brasileira de Genética, 1999. 60p. (Monografias, 9).

EBERHART, S.A.; RUSSELL, W.A. Stability parameters for comparing varieties. Crop Science, v.6, p.36-40,1966.

FINLAY, K.W.; WILKINSON, G.N. The analysis of adaptation in a plant breeding programme. Australian Journal of Agriculture Research, v.14, p.742-754, 1963.

GOMES, F.P. Curso de estatística experimental. 13.ed. Piracicaba: Livraria Nobel, 1990. 467p.

HUEHN, M. Nonparametric measures of phenotypic stability. Part I: theory. Euphytica. v.47, p.189-194, 1990.

LIN, C.S.; BINNS, M.R. A superiority measure of cultivar performance for cultivar x location data. Canadian Journal of Plant Science, v.68, p.193-198, 1988.

MOHAMMADI, R. et al. Interpreting genotype $\times$ environment interactions for durum wheat grain yields using nonparametric methods. Euphytica, v.157, p.239-251, 2007.
MOHAMMADI, R.; AMRI, A. Comparison of parametric and non-parametric methods for selecting stable and adapted durum wheat genotypes in variable environments. Euphytica, v.159, p.419-432, 2008.

MURAKAMI, D.M. et al. Considerações sobre duas metodologias de análise de estabilidade e adaptabilidade. Ciência Rural, v.34, p.71-78, 2004.

PERECIN, D.; CARGNELUTTI FILHO, A. Efeitos por comparações e por experimento em interações de experimentos fatoriais. Ciência e Agrotecnologia, v.32, p.68-72, 2008.

PLAISTED, R.L.; PETERSON, L.C. A technique for evaluating the ability of selections to yield consistently in different locations and seasons. American Potato Journal, v.36, p.381-385, 1959 .

ROSSE, L.N. et al. Comparação de métodos de regressão para avaliar a estabilidade fenotípica em cana-de-açúcar. Pesquisa Agropecuária Brasileira, v.37, p.25-32, 2002.

SABAGHNIA, N. et al. Nonparametric methods for interpreting genotype environment interaction of Lentil genotypes. Crop Science, v.46, p.1100-1106, 2006

SCAPIM, C.A. et al. Yield stability in maize (Zea mays L.) and correlations among the parameters of the Eberhart and Russell, Lin and Binns and Huehn models. Genetics and Molecular Biology, v.23, p.387-393, 2000 .

SILVA, J.G.C.; BARRETO, J.N. Aplicação da regressão linear segmentada em estudos da interação genótipo $\mathrm{x}$ ambiente. In: SIMPÓSIO DE ESTATÍSTICA APLICADA À EXPERIMENTAÇÃO AGRONÔMIVA, 1985, Campinas. Anais... Campinas: Fundação Cargil, 1985. p.49-50.

SILVA, W.C.J.; DUARTE, J.B. Métodos estatísticos para estudo de adaptabilidade e estabilidade fenotípica em soja. Pesquisa Agropecuária Brasileira, v.41, p.23-30, 2006

SOUZA, V.Q. et al. Consistency of two stability analysis methods in potatoes. Ciência Rural, v.37, p.656-661, 2007.

TAI, G.C.C. Genotype stability analysis and its application to potato regional trials. Crop Science, v.11, p.184-190, 1971.

TOLER, J.E. Patterns of genotypic performance over environmental arrays. 1990. 154f. Thesis (Ph.D.) Clemson University, South Carolina, USA.

UNIVERSIDADE FEDERAL DE LAVRAS. Estabilidade (software): versão 3.0. Lavras: UFLA-DEX, 2000. Acesso em: 15 de setembro de 2007. On line. Disponível em: http:// www.dex.ufla.br/ danielff/softwares.htm.

WRICKE, G. Zur Berechning der okovalenz bei sommerweizen und hafer. Zeitschrift fur Pflanzenzuchtung, v.52, p.127$138,1965$.

YATES, F.; COCHRAN, W.G. The analysis of group of experiments. Journal Agriculture Science. v.28, p.556580,1938

ZOBEL, R.W. et al. Statistical analysis of a yield trial. Agronomy Journal, v.80, p.388-393, 1988. 